\title{
Causality and stability for Lorentz-CPT violating electrodynamics with dimension-5 operators
}

\author{
C. Marat Reyes* \\ Departamento de Física, Universidad Autónoma Metropolitana Iztapalapa, \\ San Rafael Atlixco 186, C.P. 09340, México D.F., México, \\ and Departamento de Fúsica, Universidad de Santiago de Chile, Casilla 307, Santiago, Chile
}

(Dated: December 2010; published as Phys. Rev. D 82, 125036 (2010))

\begin{abstract}
Stringent limits on the Myers-Pospelov timelike parameter for photons $\xi<10^{-15}$ coming from astrophysical tests suggest exploring more general preferred backgrounds, such as spacelike and lightlike. We take some steps in this direction. We allow the external four vector $n$ characterizing the Lorentz symmetry breaking to have arbitrary directions in spacetime. We study the consistency for the effective field theories in each privileged frame by demanding causality, stability and analyticity. When specializing to a timelike background we found runaway solutions leading to causality and unitarity violations. We show that the lightlike theory is a higher-derivative theory with more degrees of freedom and nonanalytic solutions leading to instabilities when interactions are turned on. We demonstrate by explicit calculation that both stability and analyticity are preserved for the purely spacelike case while microcausality is highly suppressed. This new anisotropic model opens the possibility to play a role in the search for Planck-scale effects.
\end{abstract}

PACS numbers: 11.10.Lm, 11.15.-q, 11.30.Cp

\section{INTRODUCTION}

The possibility of Lorentz and CPT symmetry violation has been actively explored both theoretically and experimentally in the past recent years. Much of the motivation for considering such possibility come from the vision that spacetime at the Planck scales may depart drastically from its continuum description leaving some evidence at low energies, idea that has been reinforced by several candidate fundamental theories. The possible observability in a form of Lorentz violation has also been center of many interests for providing a route to realistic experimental scenarios where to test fundamental physics.

Searches for Lorentz and CPT violation have been performed in the following contexts: string theory [1, 2], standard model extension [3], spacetime foam [4], loop quantum gravity [5], non commutative geometry [6], modified dispersion relations [7], cosmologically varying scalars [8], higher derivative field theory [9, 10], ultraviolet regulators 11], gravity [12], nonlinear electrodynamics [13]. The range of their predictions includes all matter sectors and gravity and have been probed by a large number of experimental tests, see the tables in Ref. 14]. At present the best constraints, coming from astrophysical observational tests, are imposed on photons which also violate discrete CPT symmetry. For example, from vacuum birefringence one has the bound $k_{\Delta F}^{(3)}<2 \times 10^{-42}$ $\mathrm{GeV}$ for the Chern-Simons parameter [15, 16] and from ultra high energy cosmic rays $\xi<10^{-15}$ for the timelike parameter in the Myers and Pospelov model [17, 18]. Given such small numbers they are hardly justified for

*Electronic mail: carlos.reyesm@usach.cl
Lorentz violating corrections induced from the Planckscale and therefore for all phenomenological purposes they can be set to zero. From this consideration, it has been suggested to extend the Myers and Pospelov model to include more general backgrounds [19].

In the present work we study Lorentz violations in the framework of effective field theory with the extra ingredient to be incorporated by means of higher dimensional operators. Typically one has a modified quadratic Lagrangian density where some Lorentz violating background tensors are contracted with an operator. As formulated in the standard model extension [3], these background tensors can be viewed to arise from spontaneous symmetry breaking taking place in an underlying theory. Higher dimensional operators have become more popular in the description of Lorentz violation in the last years. One example is the Myers and Pospelov model where the Lorentz violation is incorporated via a fixed four vector pointing only in the time direction and coupled to a dimension- 5 operator. It has produced bounds from astrophysical observations 17, 18, synchrotron radiation 20], radiative corrections 21, 22], and laboratory tests [23]. Recently an extension for the photon sector including arbitrary mass dimension operators has also been constructed [16].

The incorporation of higher dimensional operators present some challenges and some caution has to be taken. These theories have been questioned for different reasons and many of the problems can be traced back to the bottomless of the Hamiltonian. That is, usually the theory has an unbound negative energy part which under interactions can couple leading to instabilities. On other hand, solutions of the plane wave ansatz can depend non analytically on the perturbative parameter, therefore undermining the validity of the effective theory. The use of higher dimensional operators not necessarily represent 
these problems, but a comprehensive study under which conditions the effective theory is consistent needs to be performed. In this work we take some steps in this direction. We perform a systematic study of the causal, stability and analytical behavior of the effective theories that introduce dimension-5 operators. We focus on the Myers-Pospelov modified theory of electrodynamics allowing to consider an arbitrary symmetry breaking four vector.

The outline of this paper is as follows. In Sec II, we introduce the Myers-Pospelov model of electrodynamics with an arbitrary spontaneous symmetry breakdown direction. The field equations and the dispersion relation are obtained. In Sec III, we analyze the propagation properties for the isotropic and anisotropic cases, and we emphasize the nonanalyticity and instabilities for the lightlike case. In Sec IV, we derive the retarded Green functions in the Coulomb gauge. Sec $\mathrm{V}$ is concerned with the quantum field theory, we obtain the propagator in the Lorentz gauge and we study microcausality for the purely spacelike case.

\section{MYERS-POSPELOV ELECTRODYNAMICS IN ARBITRARY BACKGROUNDS}

Consider the Maxwell action in the presence of a current $j_{\mu}$ plus the Myers and Pospelov term

$$
\begin{aligned}
S= & \int d^{4} x\left(-\frac{1}{4} F_{\mu \nu} F^{\mu \nu}-4 \pi j_{\mu} A^{\mu}\right. \\
& \left.+\frac{\xi}{M_{P}} n^{\mu} F_{\mu \nu}(n \cdot \partial) n_{\alpha} \widetilde{F}^{\alpha \nu}\right),
\end{aligned}
$$

where $n$ is an external four vector defining the type of background and fixed once for all, $\xi$ is a dimensionless parameter, $M_{P}$ the Planck mass, $F_{\mu \nu}$ the electromagnetic field strength tensor and $\widetilde{F}^{\alpha \beta}=\frac{1}{2} \epsilon^{\alpha \beta \rho \lambda} F_{\rho \lambda}$ its dual [9].

In its original proposal by Myers and Pospelov the action has the form

$$
S_{(5)}=\frac{\xi}{M_{P}} \int d^{4} x \epsilon^{i j k} \dot{A}_{i} \partial_{j} \dot{A}_{k}
$$

resulting from choosing the external four vector in the purely timelike direction [9]. This action and its corresponding dispersion relation has been the starting point for numerous phenomenological searches [18]. In the following we will extend the above treatment to include more general backgrounds thus considering the general cases for $n$ incorporating the spacelike and lightlike cases.

Consider the full Myers-Pospelov action re-expressed as

$$
S_{(5)}=-\frac{g}{2} \int d^{4} x \epsilon^{\mu \nu \lambda \sigma} n_{\mu} A_{\nu}(n \cdot \partial)^{2} F_{\lambda \sigma}
$$

where we have defined $g=\xi / M_{P}$. We observe the resemblance of this expression with the CPT-odd Chern
Simons term [15, 24, 25]. This can be better seen by using the notation of the recent standard model extension including nonrenormalizable operators [16], where one has

$$
\begin{gathered}
\left(k_{A F}^{(5)}\right)_{\mu}=-g(n \cdot \partial)^{2} n_{\mu}, \\
\left(k_{A F}^{(3)}\right)_{\mu}=m n_{\mu} / 2
\end{gathered}
$$

for the Myers-Pospelov and Chern-Simons modifications respectively. It can be useful to note that the Myers and Pospelov parameter is obtained from the replacement $m \rightarrow-2 g(n \cdot \partial)^{2}$.

The field equations for the action (1) are

$$
\partial_{\mu} F^{\mu \nu}+g \epsilon^{\nu \alpha \lambda \sigma} n_{\alpha}(n \cdot \partial)^{2} F_{\lambda \sigma}=4 \pi j^{\nu},
$$

which can be rewritten as

$$
\partial_{\mu} G^{\mu \nu}=4 \pi j^{\nu}
$$

where

$$
G^{\mu \nu}=F^{\mu \nu}+2 g \epsilon^{\mu \nu \alpha \beta} n_{\alpha}(n \cdot \partial)^{2} A_{\beta} .
$$

By introducing the notation $A^{\mu}=\left(A^{0}, \mathbf{A}=A^{i}\right)$, and the conventions $\eta_{\mu \nu}=\operatorname{diag}(1,-1,-1,-1), \epsilon^{0123}=\epsilon^{123}=1$ we can write the electric and magnetic fields as

$$
F_{0 i}=E^{i}, \quad F_{i j}=-\epsilon^{i j k} B^{k},
$$

or in vectorial notation

$$
\begin{gathered}
\mathbf{E}=-\frac{\partial \mathbf{A}}{\partial t}-\nabla A_{0}, \\
\mathbf{B}=\nabla \times \mathbf{A} .
\end{gathered}
$$

In terms of the physical fields for a general $n=\left(n_{0}, \mathbf{n}\right)$ we have the field equations

$$
\begin{gathered}
\nabla \cdot \mathbf{E}+2 g(n \cdot \partial)^{2}(\mathbf{n} \cdot \mathbf{B})=4 \pi \rho \\
-\frac{\partial \mathbf{E}}{\partial t}+\nabla \times \mathbf{B}+2 g(n \cdot \partial)^{2}\left(n_{0} \mathbf{B}-(\mathbf{n} \times \mathbf{E})\right)=4 \pi \mathbf{j}
\end{gathered}
$$

together with the usual homogeneous ones

$$
\nabla \cdot \mathbf{B}=0, \quad \nabla \times \mathbf{E}+\frac{\partial \mathbf{B}}{\partial t}=0 .
$$

Using the equations (13) and the last of (14) and in the absence of sources, we have

$$
\begin{aligned}
\square \mathbf{E}+ & \nabla(\nabla \cdot \mathbf{E})+2 g(n \cdot \partial)^{2} \\
& \times\left(n_{0}(\nabla \times \mathbf{E})+\frac{\partial}{\partial t}(\mathbf{n} \times \mathbf{E})\right)=0 .
\end{aligned}
$$


In order to find the dispersion relation we pass to momentum space by considering the ansatz $\mathbf{E}(x)=\widetilde{\mathbf{E}}(k) e^{-i k \cdot x}$. Replacing above, we obtain

$$
\begin{aligned}
k^{2} \widetilde{\mathbf{E}}+ & \mathbf{k}(\mathbf{k} \cdot \widetilde{\mathbf{E}})+2 i g(n \cdot k)^{2} \\
& \times\left(n_{0}(\mathbf{k} \times \widetilde{\mathbf{E}})-k_{0}(\mathbf{n} \times \widetilde{\mathbf{E}})\right)=0,
\end{aligned}
$$

and solving for the determinant gives us the Myers and Pospelov covariant dispersion relation

$$
\left(k^{2}\right)^{2}-4 g^{2}(n \cdot k)^{4}\left((k \cdot n)^{2}-k^{2} n^{2}\right)=0,
$$

which will be crucial for the rest of the work.

\section{DISPERSION RELATIONS AND ENERGY STABILITY}

In this section we study the solutions to the dispersion relation (17) when $n$ is chosen to be purely timelike, purely spacelike and lightlike. For each of the resulting effective theories we carry out an analysis over the necessary conditions for stability, causality and analyticity.

\section{A. An additional criteria: Analyticity}

Effective field theories that incorporate higher dimensional operators to describe perturbative corrections to a conventional field theory may present some drawbacks. In particular those that include dimension-5 operators as the ones we are interested in here. First, the higherderivative theory may have unbounded energy from below leading to instabilities problems when interactions come into play. Second, even the slightest inclusion of higher-derivative terms can produce an increase in the degrees of freedom with respect to the unperturbed theory. Both the negative energies and the increase of degrees of freedom are somehow related. It turns out that in general the degrees of freedom that have been incremented are also responsible for the appearance of negative energies. Moreover, this class of solutions have nonanalytical behavior in the perturbative parameter, that is, they tend to infinity when the perturbative parameters are taken to zero. Some perturbative methods have been developed in order to eliminate these additional degrees of freedom. The resulting effective theories are shown to have positive and hermitian Hamiltonians [26]. In the next subsection, we consider the analytical criteria also called perturbative constraint [27] in order to discriminate whether a theory is a higher derivative theory or not.

\section{B. The isotropic model}

We start considering the isotropic four-vector $n=$ $\left(n_{0}, 0,0,0\right)$ for which the dispersion relation (17) has the

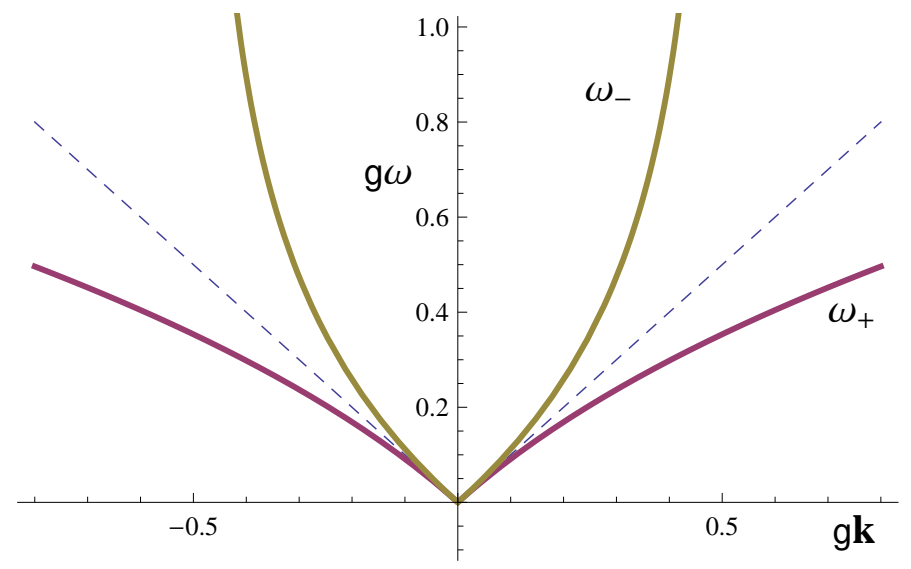

FIG. 1: The dispersion relation (19) for a purely timelike four vector $n=(1,0,0,0)$ with corresponding curves $\omega_{-}$and $\omega_{+}$ and the dashed line corresponding to the light cone.

form

$$
\left(k^{2}\right)^{2}-4 g^{2} k_{0}^{4} \mathbf{k}^{2} n_{0}^{6}=0 .
$$

Solving we obtain the frequency solutions

$$
\omega_{\lambda}(\mathbf{k})=\frac{|\mathbf{k}|}{\sqrt{1+2 g \lambda|\mathbf{k}| n_{0}^{3}}},
$$

with the two polarizations $\lambda= \pm$. The solutions correctly reproduces the usual ones in the limit $g \rightarrow 0$ and coincide with those previously derived in [20].

For momenta $|\mathbf{k}|<1 /\left(2 g n_{0}^{3}\right)$ the approximation of (19)

$$
\omega_{\lambda}(\mathbf{k}) \approx|\mathbf{k}|-g \lambda n_{0}^{3}|\mathbf{k}|^{2},
$$

gives the cubic modifications reported in [9]. For higher momenta the approximation is no longer valid for $\omega_{-}$ and its imaginary part leads to the loss of unitarity and to instabilities. This signals the need of an ultraviolet cut-off function in order to avoid runaway solutions. The quantization of this model restricted to a region of momenta $|\mathbf{k}|<|\mathbf{k}|_{\max }=1 /\left(2 g n_{0}^{3}\right)$ and using Pauli-Villars regularization methods has been performed in [21].

To discuss stability and causality we plot the dispersion relation (19) in Fig. 1 and consider the necessary conditions discussed in [28]. For $k_{+}=\left(\omega_{+}, \mathbf{k}\right)$ we observe the possibility to have negative energy by performing an observer Lorentz transformation to a boosted frame in which $\omega_{+}$is negative. For $n_{0}=1$ and fixed momentum this occurs when $1 / \sqrt{1+2 g|\mathbf{k}|}<|\mathbf{v}|<1$ and together with the maximum allowed momentum $|\mathbf{k}|_{\max }$ this leads to the requirement that the allowed concordant frames [28] in which the quantization will remain consistent are such that the boost velocity restrict to $\beta<1 / \sqrt{2}$ with respect to the rest frame.

Consider the group velocity

$$
v_{g^{ \pm}}(\mathbf{k})=\frac{\left(1 \pm g|\mathbf{k}| n_{0}^{3}\right)}{\left(1 \pm 2 g|\mathbf{k}| n_{0}^{3}\right)^{3 / 2}}
$$


and note that $v_{g^{-}}(\mathbf{k})$ can exceed the speed of light introducing problems of causality. In the next sections we compute the retarded Green function which gives us a more transparent way to analyze causality violations in the presence of interactions.

\section{The spatially anisotropic model}

For a generic external four vector $n=\left(n_{0}, \mathbf{n}\right)$ the dispersion relation (17) will be a sixth order polynomial with some roots having imaginary parts. Also, the high order character of the equation makes technically difficult to obtain the exact solutions. To simplify we will restrict to the type of anisotropies introduced by lightlike and purely spacelike preferred vectors.

Consider first the lightlike case $n=\left(n_{0}, \mathbf{n}\right)$ with $n^{2}=$ 0 , for which the dispersion relation is

$$
k_{0}^{2}-\mathbf{k}^{2}+2 g \lambda\left(n_{0} k_{0}-|\mathbf{k}||\mathbf{n}| \cos \theta\right)^{3}=0
$$

The solutions are

$$
\begin{aligned}
& \omega_{1}(\lambda, \mathbf{k})=\frac{\lambda}{6 g|\mathbf{n}|^{3}}\left(6 g \lambda z \cos \theta-1+\frac{(1-12 g \lambda z \cos \theta)}{\Delta^{1 / 3}}+\Delta^{1 / 3}\right), \\
& \omega_{2}(\lambda, \mathbf{k})=\frac{\lambda}{6 g|\mathbf{n}|^{3}}\left(6 g \lambda z \cos \theta-1-\frac{(1+i \sqrt{3})(1-12 g \lambda z \cos \theta)}{2 \Delta^{1 / 3}}-\frac{(1-i \sqrt{3}) \Delta^{1 / 3}}{2}\right), \\
& \omega_{3}(\lambda, \mathbf{k})=\frac{\lambda}{6 g|\mathbf{n}|^{3}}\left(6 g \lambda z \cos \theta-1-\frac{(1-i \sqrt{3})(1-12 g \lambda z \cos \theta)}{2 \Delta^{1 / 3}}-\frac{(1+i \sqrt{3}) \Delta^{1 / 3}}{2}\right),
\end{aligned}
$$

where

$$
\Delta(\lambda, z)=\Delta_{1}(\lambda, z)+\Delta_{2}(\lambda, z),
$$

and

$$
\begin{aligned}
\Delta_{1}(\lambda, z)= & -1+54 g^{2} z^{2}+18 g \lambda z \cos \theta-54 g^{2} z^{2} \cos ^{2} \theta, \\
\Delta_{2}(\lambda, z)= & \left((-1+12 g \lambda z \cos \theta)^{3}+(1-18 g \lambda z \cos \theta\right. \\
& \left.\left.-27 g^{2} z^{2}(1-2 \cos (2 \theta))\right)^{2}\right)^{1 / 2} .
\end{aligned}
$$

Above we have made explicit the relation $n^{2}=0$, defined $z=|\mathbf{k}||\mathbf{n}|^{3}$ and considered $\theta$ the angle between $\mathbf{k}$ and $\mathbf{n}$.

Consider the approximations of small $g$ at linear order:

$$
\begin{aligned}
\omega_{1,3}(\lambda, \mathbf{k}) & \approx \lambda|\mathbf{k}|+\mathcal{O}(g), \\
\omega_{2}(\lambda, \mathbf{k}) & \approx \frac{\lambda}{2 g|\mathbf{n}|^{3}}+3|\mathbf{k}| \cos \theta+\mathcal{O}(g) .
\end{aligned}
$$

We have two solutions that approximate well the usual solutions when taking the limit $g \rightarrow 0$ and one extra degree of freedom that is nonanalytic in the perturbative parameter. We expect the lightlike theory to develop negative energies and have nonunitarity evolution.

For the case where $n$ is purely spatial $n=(0, \mathbf{n})$ we have

$$
\left(k^{2}\right)^{2}-4 g^{2}(\mathbf{k} \cdot \mathbf{n})^{4}\left((\mathbf{k} \cdot \mathbf{n})^{2}+\left(k_{0}^{2}-\mathbf{k}^{2}\right) \mathbf{n}^{2}\right)=0,
$$

with roots

$$
\begin{aligned}
\omega_{ \pm}(\mathbf{k})= & \left(\mathbf{k}^{2}+2 g^{2} \mathbf{n}^{2}(\mathbf{k} \cdot \mathbf{n})^{4}\right. \\
& \left. \pm 2 g|\mathbf{k} \cdot \mathbf{n}|^{3} \sqrt{1+g^{2} \mathbf{n}^{4}(\mathbf{k} \cdot \mathbf{n})^{2}}\right)^{1 / 2} .
\end{aligned}
$$

For small momenta we have at leading order

$$
\omega_{\lambda}(\mathbf{k}) \approx|\mathbf{k}|-g \lambda|\mathbf{n}|^{3}|\cos \theta|^{3}|\mathbf{k}|^{2},
$$

where again $\theta$ is the angle between $\mathbf{k}$ and $\mathbf{n}$. The above expression is very similar to the one given in Eq (20), but valid for all momenta.

The group and phase velocities are, respectively

$$
v_{g^{ \pm}}=\frac{1+4 g^{2}|\mathbf{k}|^{2} \cos ^{4} \theta \pm g|\mathbf{k}||\cos \theta|^{3}\left(3+4 g^{2}|\mathbf{k}|^{2} \cos ^{2} \theta\right) / \sqrt{1+g^{2}|\mathbf{k}|^{2} \cos ^{2} \theta}}{\left(1+2 g^{2}|\mathbf{k}|^{2} \cos ^{4} \theta \pm 2 g|\mathbf{k}||\cos \theta|^{3} \sqrt{1+g^{2}|\mathbf{k}|^{2} \cos ^{2} \theta}\right)^{1 / 2}}
$$

and 


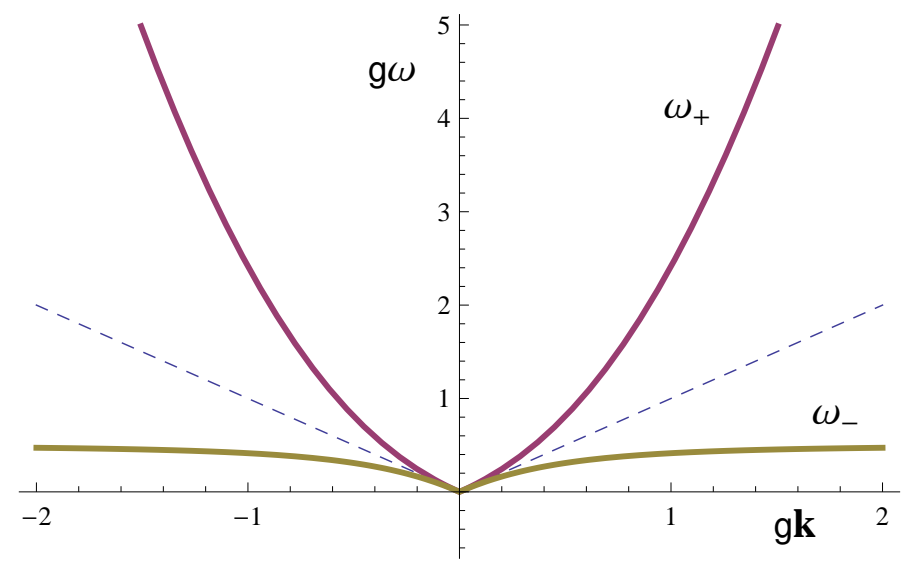

FIG. 2: Dispersion relation (29) for the case (b) with $\mathbf{n}^{2}=$ 1 with corresponding curves $\omega_{+}$and $\omega_{-}$and the light cone (dashed).

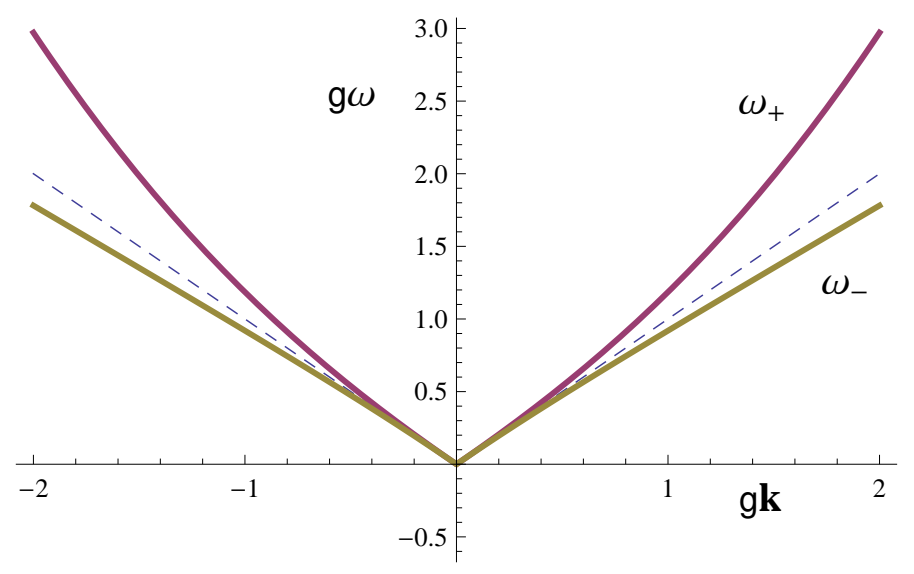

FIG. 3: Dispersion relation (29) for the case (c) with $\cos \theta=$ $1 / 2$ and $\mathbf{n}^{2}=1$ with corresponding curves $\omega_{+}$and $\omega_{-}$and the light cone (dashed).

We can distinguish three cases for the dispersion relation (29):

(a) when $\mathbf{k}$ and $\mathbf{n}$ are perpendicular,

(b) parallel or anti-parallel with $|\cos \theta|=1$,

(c) directions such that $|\cos \theta|<1$. For the case (a) we recover the usual dispersion relation $\omega=|\mathbf{k}|$, also mentioned in [19] as a blind direction. To analyze the cases (b) and (c) consider the plots Fig. 2 and Fig. 3.

For the cases (b) and (c) the momentum $k_{-}=\left(\omega_{-}, k\right)$ is outside the light cone which threatens the stability of the theory. Therefore for small momentum we must restrict to the region

$$
\beta_{\lambda}<1-\lambda g|\mathbf{k}||\cos \theta|^{3}
$$

in order to have concordant frames. This includes a large number of concordant frames since the boost velocity is very near the light cone. Going to large momenta we have that $\omega_{-}$approaches the constant value $\omega_{-}(\mathbf{k}) \approx \frac{1}{2 g}$ for the case $(b)$ and it diverges as $\omega_{-}(\mathbf{k}) \approx \sin \theta|\mathbf{k}|$ for the case (c) and therefore the velocity group approaches the constant value $v_{g^{-}}(\mathbf{k}) \approx \sin \theta$. In consequence any finite boost will introduce instabilities in the parallel or antiparallel directions for high momentum. We expect to have more concordant frames for propagations approaching the perpendicular direction.

On the other hand we see that $v_{g^{+}}(\mathbf{k})$ can exceed the speed of light which indicates possible violations of microcausality.

\section{CAUSALITY AND RETARDED GREEN FUNCTIONS}

In this section to study the causal behavior of the gauge fields in the presence of interactions we compute the retarded Green function. As standard we assume a delta source interaction and to avoid to deal with nonphysical degrees of freedom we work in the Coulomb gauge $\nabla \cdot \mathbf{A}=0$.

\section{A. Transverse Green functions}

The transverse Green function will be constructed by first reducing the degree of freedom corresponding to $A_{0}$. Hence, from (10) and (12) we replace the non dynamical relation

$$
A_{0}=2 g \frac{(n \cdot \partial)^{2}}{\nabla^{2}}(\mathbf{n} \cdot \mathbf{B})-4 \pi \frac{1}{\nabla^{2}} \rho
$$

in (13) to yield the expression

$$
\begin{aligned}
& \square \mathbf{A}+2 g \frac{(n \cdot \partial)^{2}}{\nabla^{2}} \nabla\left(\mathbf{n} \cdot \nabla \times \frac{\partial \mathbf{A}}{\partial t}\right)+2 g(n \cdot \partial)^{2} \\
& \times\left(n_{0} \nabla \times \mathbf{A}+\mathbf{n} \times \frac{\partial \mathbf{A}}{\partial t}\right) \\
& +4 g^{2} \frac{(n \cdot \partial)^{4}}{\nabla^{2}} \mathbf{n} \times \nabla(\mathbf{n} \cdot \nabla \times \mathbf{A})=4 \pi \mathbf{J}_{T}
\end{aligned}
$$

Above we have used current conservation $\partial \rho / \partial t+\nabla \cdot \mathbf{j}=0$ and defined the transverse current $\mathbf{J}_{T}$

$$
\mathbf{J}_{T}=\mathbf{j}-\frac{\nabla(\nabla \cdot \mathbf{j})}{\nabla^{2}}+2 g \frac{(n \cdot \partial)^{2}}{\nabla^{2}} \mathbf{n} \times \nabla \rho,
$$

which can be easily checked to satisfy the conserved equation $\nabla \cdot \mathbf{J}_{T}=0$. Now, provided the gauge field is transverse we have

$$
\begin{aligned}
\mathbf{n} \times \nabla(\mathbf{n} \cdot \nabla \times \mathbf{A})= & \left(\mathbf{n}^{2} \nabla^{2}-(\mathbf{n} \cdot \nabla)^{2}\right) \mathbf{A} \\
& +\left((\mathbf{n} \cdot \nabla) \nabla-\mathbf{n} \nabla^{2}\right)(\mathbf{n} \cdot \mathbf{A})
\end{aligned}
$$


which allows us to rewrite the last term in (35) and so obtain

$$
\begin{aligned}
& \left(\square+4 g^{2}(n \cdot \partial)^{4}\left(\mathbf{n}^{2}-\frac{(\mathbf{n} \cdot \nabla)^{2}}{\nabla^{2}}\right)\right) \mathbf{A}+2 g \frac{(n \cdot \partial)^{2}}{\nabla^{2}} \\
& \times \nabla\left(\mathbf{n} \cdot \nabla \times \frac{\partial \mathbf{A}}{\partial t}\right)+2 g(n \cdot \partial)^{2}\left(n_{0} \nabla \times \mathbf{A}+\mathbf{n} \times \frac{\partial \mathbf{A}}{\partial t}\right) \\
& +4 g^{2}(n \cdot \partial)^{4}\left(\frac{\nabla(\mathbf{n} \cdot \nabla)}{\nabla^{2}}-\mathbf{n}\right)(\mathbf{n} \cdot \mathbf{A})=4 \pi \mathbf{J}_{T} .
\end{aligned}
$$

Taking components of the above equation of motion we have

$$
M_{i j}\left(\partial_{0}, \nabla\right) A^{j}=J_{T}^{i},
$$

where

$$
\begin{aligned}
& M_{i j}\left(\partial_{0}, \nabla\right) \\
= & {\left[\left(\square+4 g^{2}(n \cdot \partial)^{4}\left(\mathbf{n}^{2}-\frac{(\mathbf{n} \cdot \nabla)^{2}}{\nabla^{2}}\right)\right) \delta_{i j}\right.} \\
& +2 g(n \cdot \partial)^{2}\left(n_{0} \epsilon^{i l j} \partial_{l}+\partial_{0}\left(\epsilon^{i l j} n^{l}+\frac{\partial_{i} \partial_{m}}{\nabla^{2}} \epsilon^{l m j} n^{l}\right)\right) \\
& \left.+4 g^{2}(n \cdot \partial)^{4}\left(\frac{\partial_{i} \partial_{l} n^{l}}{\nabla^{2}}-n^{i}\right) n^{j}\right] .
\end{aligned}
$$

After some straightforward calculation the transverse retarded Green function is given by

$$
G_{j k}(x-y)=T_{j k}\left(\partial_{0}, \nabla\right) D(x-y),
$$

where the tensor

$$
T_{j k}\left(\partial_{0}, \nabla\right)=\pi_{j k} \square+4 g^{2}(n \cdot \partial)^{4} s_{j k}+2 g(n \cdot \partial)^{2} a_{j k},
$$

is given in terms of the operators

$$
\begin{gathered}
\pi_{j k}=\left(\delta_{j k}-\frac{\partial_{j} \partial_{k}}{\nabla^{2}}\right), \\
s_{j k}=\left(n^{j}-\frac{(\mathbf{n} \cdot \nabla)}{\nabla^{2}} \partial_{j}\right)\left(n^{k}-\frac{(\mathbf{n} \cdot \nabla)}{\nabla^{2}} \partial_{k}\right), \\
a_{j k}=\left(n_{0}+\frac{(\mathbf{n} \cdot \nabla)}{\nabla^{2}} \partial_{0}\right) \epsilon^{j k l} \partial_{l},
\end{gathered}
$$

The scalar retarded Green function $D(x-y)$ is defined to satisfy

$$
\left(\square^{2}+4 g^{2}(n \cdot \partial)^{4}\left((n \cdot \partial)^{2}-n^{2} \square\right)\right) D(x-y)=4 \pi \delta(x-y) .
$$

The fourier transform lead us to the integral

$$
\begin{aligned}
& D(x-y)=\frac{4 \pi}{(2 \pi)^{4}} \int_{C} d^{4} k \\
& \times \frac{e^{-i k \cdot(x-y)}}{\left(k^{2}\right)^{2}-4 g^{2}(n \cdot k)^{4}\left((k \cdot n)^{2}-k^{2} n^{2}\right)},
\end{aligned}
$$

where the contour of the curve $C$, in the complex $k_{0}$ plane, defines the boundary conditions to be imposed on the various Green functions. For the retarded Green function the curve is defined above the real axis and is denoted by $C_{R}$. The corresponding scalar function $D_{R}(x-y)$ controls the causal behavior of the theory and therefore to study causality will be enough to compute $D_{R}(x-y)$.

\section{B. Causality for $n$ purely spacelike}

For the case $n=(0, \mathbf{n})$ the integral (47) is

$$
D_{R}(z)=\frac{4 \pi}{(2 \pi)^{4}} \int d^{3} x e^{i \mathbf{k} \cdot \mathbf{z}} \int_{C_{R}} d k_{0} \frac{e^{-i k_{0} z_{0}}}{\left(k_{0}^{2}-\omega_{+}^{2}\right)\left(k_{0}^{2}-\omega_{-}^{2}\right)},
$$

where $z=x-y$ and the solutions $\omega_{ \pm}$are given by (29).

We observe that the integral

$$
I_{R}\left(z_{0}\right)=\int_{C_{R}} d k_{0} \frac{e^{-i k_{0} z_{0}}}{\left(k_{0}^{2}-\omega_{+}^{2}\right)\left(k_{0}^{2}-\omega_{-}^{2}\right)},
$$

vanishes for $z_{0}<0$ since the contour $C_{R}$ must be closed in the upper half plane therefore failing to enclose any of the poles $\omega_{ \pm}$lying on the real axis. We have in consequence that causality is preserved for the spacelike background.

\section{Causality for $n$ purely timelike}

Consider now $n=(1,0,0,0)$, we have from (47)

$$
\begin{aligned}
D_{R}(x)= & \frac{4 \pi}{(2 \pi)^{4}} \int d^{3} k \frac{e^{i \mathbf{k} \cdot \mathbf{x}}}{\left(1-4 g^{2}|\mathbf{k}|^{2}\right)} \\
& \times \int_{C_{R}} d k_{0} \frac{e^{-i k_{0} x_{0}}}{\left(k_{0}^{2}-\omega_{+}^{2}\right)\left(k_{0}^{2}-\omega_{-}^{2}\right)},
\end{aligned}
$$

where from (19) the solutions are

$$
\omega_{+}(\mathbf{k})=\frac{|\mathbf{k}|}{\sqrt{1+2 g|\mathbf{k}|}}, \quad \omega_{-}(\mathbf{k})=\frac{|\mathbf{k}|}{\sqrt{1-2 g|\mathbf{k}|}},
$$

and we have set $y=0$.

The calculation of $D_{R}(x)$ will be done in two stages depending on the sign of $x_{0}$.

(i) For $x_{0}<0$ we focus on the contour integral

$$
I_{R}\left(x_{0}^{-}\right)=\int_{C_{R}} d k_{0} \frac{e^{i k_{0}\left|x_{0}\right|}}{\left(k_{0}^{2}-\omega_{+}^{2}\right)\left(k_{0}^{2}-\omega_{-}^{2}\right)} .
$$

We must close the contour of $C_{R}$ in the upper half plane and therefore we have contributions only when when $|\mathbf{k}|>1 /(2 g)$ due to the pole in $i\left|\omega_{-}\right|$with

$$
\left|\omega_{-}\right|=\frac{|\mathbf{k}|}{\sqrt{2 g|\mathbf{k}|-1}}
$$


The contour integral gives

$$
I_{R}\left(x_{0}^{-}\right)=\frac{-\pi e^{-\left|\omega_{-}\right|\left|x_{0}\right|}}{\left|\omega_{-}\right|\left(\omega_{+}^{2}+\left|\omega_{-}\right|^{2}\right)},
$$

and from (50) we have

$$
\begin{aligned}
D_{R}\left(x^{-}\right)= & \frac{1}{8 \pi} \int_{1 /(2 g)}^{\infty} d|\mathbf{k}| \\
& \int_{-\pi}^{\pi} \sin \theta d \theta \frac{e^{-\left|\omega_{-}\right|\left|x_{0}\right|}}{g|\mathbf{k}|\left|\omega_{-}\right|} e^{i|\mathbf{k}||\mathbf{x}| \cos \theta},
\end{aligned}
$$

where we have used

$$
\omega_{+}^{2}+\left|\omega_{-}\right|^{2}=\frac{-4 g|\mathbf{k}|^{3}}{1-4 g^{2}|\mathbf{k}|^{2}}
$$

Integrating in the angle we arrive at

$$
D_{R}\left(x^{-}\right)=\frac{1}{4 \pi g r} \int_{1 /(2 g)}^{\infty} d|\mathbf{k}| \frac{e^{-\left|\omega_{-}\right|\left|x_{0}\right|} \sin (|\mathbf{k}| r)}{|\mathbf{k}|^{2}\left|\omega_{-}\right|}
$$

where we have introduced the notation $r=|\mathbf{x}|$. In order to arrive to a more elegant expression we perform the change of variables $x=\frac{1}{|\mathbf{k}|}$ leading to

$$
\begin{aligned}
D_{R}\left(x^{-}\right)= & \frac{1}{4 \pi g r} \int_{0}^{2 g} d x e^{-\frac{\left|x_{0}\right|}{\sqrt{x(2 g-x)}}} \sin \left(\frac{r}{x}\right) \\
& \times \sqrt{x(2 g-x)}
\end{aligned}
$$

again making $z=x-g$ we have

$$
\begin{aligned}
D_{R}\left(x^{-}\right)= & \frac{1}{2 \pi g r} \int_{0}^{g} d z e^{-\frac{\left|x_{0}\right|}{\sqrt{g^{2}-z^{2}}}} \sqrt{g^{2}-z^{2}} \\
& \times \cos \left(\frac{r z}{g^{2}-z^{2}}\right) \sin \left(\frac{g r}{g^{2}-z^{2}}\right) .
\end{aligned}
$$

From the above expression one can already appreciate causality violations effects, however to end up with a closed expression for $D_{R}$ we will continue with the next case.

(ii) For $x_{0}>0$ the contour integral is

$$
I_{R}\left(x_{0}^{+}\right)=\int_{C_{R}} d k_{0} \frac{e^{-i k_{0}\left|x_{0}\right|}}{\left(k_{0}^{2}-\omega_{+}^{2}\right)\left(k_{0}^{2}-\omega_{-}^{2}\right)},
$$

which has to closed in the lower half plane. For $|\mathbf{k}|<$ $1 /(2 g)$ the integral includes the poles $\pm \omega_{+}$and $\pm \omega_{-}$and we obtain

$$
I_{R}\left(x_{0}^{+}\right)=-2 \pi\left(\frac{\sin \left(\omega_{+}\left|x_{0}\right|\right)}{\omega_{+}\left(\omega_{+}^{2}-\omega_{-}^{2}\right)}-\frac{\sin \left(\omega_{-}\left|x_{0}\right|\right)}{\omega_{-}\left(\omega_{+}^{2}-\omega_{-}^{2}\right)}\right) .
$$

For $|\mathbf{k}|>1 /(2 g)$ we have the poles $-i\left|\omega_{-}\right|$and $\pm \omega_{+}$, and we obtain

$$
\begin{aligned}
I_{R}\left(x_{0}^{+}\right)= & -2 \pi\left(\frac{\sin \left(\omega_{+}\left|x_{0}\right|\right)}{\omega_{+}\left(\omega_{+}^{2}-\omega_{-}^{2}\right)}\right. \\
& \left.+\frac{e^{-\left|\omega_{-}\right|\left|x_{0}\right|}}{2\left|\omega_{-}\right|\left(\omega_{+}^{2}+\left|\omega_{-}\right|^{2}\right)}\right),
\end{aligned}
$$

where we have introduced $\left|\omega_{-}\right|$in the second term.

Considering the first terms in (61) and (62) we have a contribution

$$
D_{R}^{(1)}\left(x^{+}\right)=\frac{1}{2 \pi g r} \int_{0}^{\infty} d|\mathbf{k}| \frac{\sin \left(\omega_{+}\left|x_{0}\right|\right) \sin (|\mathbf{k}| r)}{|\mathbf{k}|^{2} \omega_{+}},
$$

and from the second term in (61) we have

$$
\begin{aligned}
D_{R}^{(2)}\left(x^{+}\right)= & \frac{-1}{2 \pi g r} \int_{0}^{1 /(2 g)} d|\mathbf{k}| \\
& \times \frac{\sin \left(\omega_{-}\left|x_{0}\right|\right) \sin (|\mathbf{k}| r)}{|\mathbf{k}|^{2} \omega_{-}} .
\end{aligned}
$$

The third contribution coming from the second term in (62) is the same as the one in (54) and therefore we have

$$
D_{R}^{(3)}\left(x^{+}\right)=D_{R}\left(x^{-}\right) .
$$

Adding the three contributions we arrive at

$$
\begin{aligned}
D_{R}\left(x^{+}\right)= & \frac{1}{\pi g r}\left[\int_{g}^{\infty} d z \sin \left(\frac{x_{0}}{\sqrt{z^{2}-g^{2}}}\right)\right. \\
& \times \sqrt{z^{2}-g^{2}} \cos \left(\frac{z r}{z^{2}-g^{2}}\right) \sin \left(\frac{g r}{z^{2}-g^{2}}\right) \\
& +\frac{1}{2} \int_{0}^{g} d z e^{\frac{-\left|x_{0}\right|}{\sqrt{g^{2}-z^{2}}}} \sqrt{g^{2}-z^{2}} \\
& \left.\times \cos \left(\frac{z r}{g^{2}-z^{2}}\right) \sin \left(\frac{g r}{g^{2}-z^{2}}\right)\right]
\end{aligned}
$$

The total scalar retarded Green function $D_{R}(x)=$ $D_{R}\left(x^{+}\right) \theta\left(x_{0}\right)+D_{R}\left(x^{-}\right) \theta\left(-x_{0}\right)$ is given by

$$
\begin{aligned}
D_{R}(x)= & \frac{1}{\pi g r}\left[\theta\left(x_{0}\right) \int_{g}^{\infty} d z \sin \left(\frac{x_{0}}{\sqrt{z^{2}-g^{2}}}\right)\right. \\
& \times \sqrt{z^{2}-g^{2}} \cos \left(\frac{z r}{z^{2}-g^{2}}\right) \sin \left(\frac{g r}{z^{2}-g^{2}}\right) \\
& +\frac{1}{2} \int_{0}^{g} d z e^{\frac{-\left|x_{0}\right|}{\sqrt{g^{2}-z^{2}}}} \sqrt{g^{2}-z^{2}} \\
& \left.\times \cos \left(\frac{z r}{g^{2}-z^{2}}\right) \sin \left(\frac{g r}{g^{2}-z^{2}}\right)\right] .
\end{aligned}
$$

As we have mentioned there is a response of the fields before the source has acted which reveals violations of causality.

\section{THE QUANTUM FIELD THEORY}

In the next first subsection we compute the propagator in the covariant Lorentz gauge in any preferred background. In the second we compute the commutator function for a purely spacelike background giving an estimation of the microcausality violation for spacelike separations near the light cone. The purely timelike background has been studied in [21]. 


\section{A. The propagator in the Lorentz gauge}

Consider the free Lagrangian density (1)

$$
\begin{aligned}
\mathcal{L}= & -\frac{1}{4} F_{\mu \nu} F^{\mu \nu}-\frac{g}{2} n_{\mu} \epsilon^{\mu \nu \lambda \sigma} A_{\nu}(n \cdot \partial)^{2} F_{\lambda \sigma} \\
& -\frac{1}{2}\left(\partial^{\mu} A_{\mu}\right)^{2}
\end{aligned}
$$

where we have included a Lorentz gauge fixing term. We can write modulo total derivatives

$$
\mathcal{L}=\frac{1}{2} A_{\nu}\left(\eta^{\nu \sigma} \square-2 g \epsilon^{\mu \nu \lambda \sigma} n_{\mu}(n \cdot \partial)^{2} \partial_{\lambda}\right) A_{\sigma},
$$

where we identify the photon kinetic operator

$$
\left(\Delta^{-1}\right)^{\nu \sigma}=\eta^{\nu \sigma} \square-2 g \epsilon^{\mu \nu \lambda \sigma} n_{\mu}(n \cdot \partial)^{2} \partial_{\lambda} .
$$

We want to find the Feynman propagator by inverting the above operator, to this aim we go to the momentum representation considering $A_{\mu}(x)=A_{\mu}(k) e^{-i(k \cdot x)}$ to obtain

$$
\left(\Delta^{-1}\right)^{\nu \sigma}=-k^{2} \eta^{\nu \sigma}+2 i g \epsilon^{\nu \mu \lambda \sigma} n_{\mu}(n \cdot k)^{2} k_{\lambda} .
$$

The Feynman propagator resulting from the inversion is

$$
\begin{aligned}
\left(\Delta_{F}(k)\right)_{\sigma \lambda}= & \frac{1}{G}\left[-k^{2} \eta_{\sigma \lambda}+2 i g(n \cdot k)^{2} \epsilon_{\sigma \alpha \beta \lambda} n^{\alpha} k^{\beta}\right. \\
& -4 g^{2}(n \cdot k)^{4}\left(n_{\sigma} n_{\lambda}+k_{\sigma} k_{\lambda}\left(\frac{n^{2}}{k^{2}}\right)\right. \\
& \left.\left.-\left(n_{\sigma} k_{\lambda}+n_{\lambda} k_{\sigma}\right) \frac{(n \cdot k)}{k^{2}}\right)\right],
\end{aligned}
$$

with the pole structure dominated by

$$
G=\left(k^{2}\right)^{2}-4 g^{2}(n \cdot k)^{4}\left((k \cdot n)^{2}-k^{2} n^{2}\right) .
$$

The case $n=(1,0,0,0)$ correctly reproduces the one calculated in the reference [21]

$$
\begin{aligned}
\left(\Delta_{F}(k)\right)_{\mu \nu}= & \frac{1}{\left(\left(k^{2}\right)^{2}-4 g^{2} k_{0}^{4}|\mathbf{k}|^{2}\right)}\left[-k^{2} \eta_{\mu \nu}\right. \\
& +2 i g k_{0}^{2} \epsilon^{l m r} k_{m} \eta_{l \mu} \eta_{r \nu}-\frac{4 g^{2} k_{0}^{4}}{k^{2}} k_{l} k_{r} \delta_{\mu}^{l} \delta_{\nu}^{r} \\
& \left.+\frac{4 g^{2} k_{0}^{4}|\mathbf{k}|^{2}}{k^{2}} \eta_{0 \mu} \eta_{0 \nu}\right]
\end{aligned}
$$

\section{B. Microcausality}

Consider the commutator of the gauge fields

$$
\left[A_{i}(z), A_{j}(0)\right]=i T_{i j}\left(-i \partial_{0},-i \nabla\right) D(z),
$$

where the tensor $T_{i j}\left(-i \partial_{0},-i \nabla\right)$ is given by the expression (42) and recall from Eq. (47) the scalar Green function

$$
D(z)=\frac{4 \pi}{(2 \pi)^{4}} \oint_{C} d^{4} k \frac{e^{-i k \cdot z}}{\left(k^{2}\right)^{2}-4 g^{2}(n \cdot k)^{4}\left((k \cdot n)^{2}-k^{2} n^{2}\right)} .
$$

A few observations are in order. The non locality of the tensor $T_{i j}$ may introduce apparent microcausality violations in the commutator (75) which can be bypassed by considering physical fields such as the electric and magnetic fields. Therefore, in the following we will consider the commutator (75) involving only physical fields which amounts to introduce more derivatives and to possibly modify the tensor structure of $T_{i j}$. We stress that the scalar Green function the function relevant for the study of causality of the theory remains intact.

In the following to provide an estimation of microcausality violations we consider spacelike separations $z^{2}<0$. Consider the integral (76) for a purely spacelike four vector $n$

$$
D(z)=\frac{4 \pi}{(2 \pi)^{4}} \oint_{C} d^{4} k \frac{e^{-i k \cdot z}}{\left(k_{0}^{2}-\omega_{+}^{2}\right)\left(k_{0}^{2}-\omega_{-}^{2}\right)},
$$

where the solutions $\omega_{ \pm}$are given by (28). Without loss of generality we will consider $n=(0,0,0,1)$ in which case the integral (77) takes the form

$$
D(z)=\frac{4 \pi}{(2 \pi)^{4}} \oint_{C} d^{4} k \frac{e^{-i\left(k_{0} z_{0}-\mathbf{k} \cdot \mathbf{z}\right)}}{\left(k_{0}^{2}-\omega_{+}^{2}\right)\left(k_{0}^{2}-\omega_{-}^{2}\right)},
$$

with

$$
\omega_{ \pm}^{2}=k_{1}^{2}+k_{2}^{2}+\widetilde{\omega}_{ \pm}^{2}\left(k_{3}\right)
$$

and the function of $k_{3}$ given by

$$
\widetilde{\omega}_{ \pm}\left(k_{3}\right)=\sqrt{k_{3}^{2}+g^{2} k_{3}^{4}} \pm g k_{3}^{2} .
$$

Unfortunately we cannot simplify by performing a Lorentz transformation to a frame where $z_{0}=0$, as achieved in the usual case, since any finite boost would then generate a zeroth component for $n$ converting the dispersion relation into a higher order polynomial with imaginary solutions.

We can still perform a boost in the perpendicular plane in order to simplify, namely

$$
k_{0} z_{0}-\left(\mathbf{k}_{\perp} \cdot \mathbf{z}_{\perp}\right)-k_{3} z_{3}=k_{0}^{\prime} z_{0}-\left(\mathbf{k}_{\perp}^{\prime} \cdot \mathbf{z}_{\perp}\right)-k_{3} z_{3},
$$

where

$$
\begin{aligned}
k_{0}^{\prime} & =\gamma\left(k_{0}-\left(\mathbf{k}_{\perp} \cdot \mathbf{z}_{\perp}\right)\right), \\
\mathbf{k}_{\perp}^{\prime} & =\mathbf{k}_{\perp}+\left(\frac{(\gamma-1)}{v^{2}}\left(\mathbf{k}_{\perp} \cdot \mathbf{z}_{\perp}\right)-\gamma k_{0}\right) \mathbf{v} .
\end{aligned}
$$

With a suitable additional boost for $z$ we can write (78) as

$$
D\left(\bar{z}_{0}, z_{3}\right)=\frac{4 \pi}{(2 \pi)^{4}} \oint_{C} d^{4} k \frac{e^{-i\left(k_{0} \bar{z}_{0}-k_{3} z_{3}\right)}}{\left(k_{0}^{2}-\omega_{+}^{2}\right)\left(k_{0}^{2}-\omega_{-}^{2}\right)}
$$

where

$$
\bar{z}_{0}=\sqrt{\frac{z_{0}^{2}-z_{1}^{2}-z_{2}^{2}}{z_{0}^{2}}} z_{0}
$$


We note that for $z_{0}^{2}<z_{1}^{2}+z_{2}^{2}$ we can set $\bar{z}_{0}=0$ and therefore the integral (84) vanishes due to the symmetric contribution of the poles.

For $z_{0}^{2} \geq z_{1}^{2}+z_{2}^{2}$ consider the integral in the $k_{0}$ plane

$$
I\left(\bar{z}_{0}\right)=\oint_{C} d k_{0} \frac{e^{-i k_{0} \bar{z}_{0}}}{\left(k_{0}^{2}-\omega_{+}^{2}\right)\left(k_{0}^{2}-\omega_{-}^{2}\right)} .
$$

Integrating we arrive at

$$
\begin{aligned}
& D\left(\bar{z}_{0}, z_{3}\right)=\frac{4 \pi}{(2 \pi)^{3}} \int d^{3} k e^{i k_{3} z_{3}} \\
& \left(\frac{\sin \omega_{-} \bar{z}_{0}}{\omega_{-}\left(\omega_{+}^{2}-\omega_{-}^{2}\right)}-\frac{\sin \omega_{+} \bar{z}_{0}}{\omega_{+}\left(\omega_{+}^{2}-\omega_{-}^{2}\right)}\right) .
\end{aligned}
$$

We change to polar coordinates for the perpendicular variables $k_{1}$ and $k_{2}$ such that

$$
\begin{aligned}
& D\left(\bar{z}_{0}, z_{3}\right)=\frac{4 \pi}{(2 \pi)^{2}} \int_{-\infty}^{\infty} d k_{3} e^{i k_{3} z_{3}} \int_{0}^{\infty} \rho d \rho \\
& \times\left(\frac{\sin \left(\omega_{-} \bar{z}_{0}\right)}{\omega_{-}\left(\omega_{+}^{2}-\omega_{-}^{2}\right)}-\frac{\sin \left(\omega_{+} \bar{z}_{0}\right)}{\omega_{+}\left(\omega_{+}^{2}-\omega_{-}^{2}\right)}\right)
\end{aligned}
$$

where

$$
\omega_{ \pm}^{2}=\rho^{2}+\widetilde{\omega}_{ \pm}^{2}\left(k_{3}\right),
$$

Given that $\omega_{+}^{2}-\omega_{-}^{2}$ is a function of $k_{3}$ only the integral in $\rho$ is easily done arriving at

$$
\begin{aligned}
& D\left(\bar{z}_{0}, z_{3}\right)=-\frac{4 \pi}{(2 \pi)^{2} \bar{z}_{0}} \int_{-\infty}^{\infty} d k_{3} \\
& \times e^{i k_{3} z_{3}}\left(\frac{\cos \left(\widetilde{\omega}_{+} \bar{z}_{0}\right)-\cos \left(\widetilde{\omega}_{-} \bar{z}_{0}\right)}{\widetilde{\omega}_{+}^{2}-\widetilde{\omega}_{-}^{2}}\right),
\end{aligned}
$$

where we are neglected a fast oscillating part.

Let us rewrite

$$
\begin{aligned}
& D\left(\bar{z}_{0}, z_{3}\right)=-\frac{1}{8 \pi \bar{z}_{0}} \int_{-\infty}^{\infty} d k_{3} e^{i k_{3} z_{3}} \\
& \times\left(\frac{e^{i \widetilde{\omega}_{+} \bar{z}_{0}}+e^{-i \widetilde{\omega}_{+} \bar{z}_{0}}-e^{i \widetilde{\omega}_{-} \bar{z}_{0}}-e^{-i \widetilde{\omega}_{-} \bar{z}_{0}}}{g k_{3}^{2} \sqrt{k_{3}^{2}+g^{2} k_{3}^{4}}}\right),
\end{aligned}
$$

and let us define

$$
\begin{aligned}
D\left(\bar{z}_{0}, z_{3}\right)= & -\frac{1}{8 \pi \bar{z}_{0}}\left(I_{1+}\left(\bar{z}_{0}\right)\right. \\
& \left.+I_{1-}\left(\bar{z}_{0}\right)-I_{2+}\left(\bar{z}_{0}\right)-I_{2-}\left(\bar{z}_{0}\right)\right) .
\end{aligned}
$$

The integrals that shall be computed have the form

$$
I_{a \lambda}\left(\bar{z}_{0}\right)=\int_{-\infty}^{\infty} d k_{3} f\left(k_{3}\right) e^{i \Phi_{a \lambda}\left(k_{3}\right)},
$$

with the phases being

$$
\Phi_{a \lambda}\left(k_{3}\right)=k_{3} z_{3}+\lambda \bar{z}_{0}\left(\sqrt{k_{3}^{2}+g^{2} k_{3}^{4}}-(-1)^{a} g k_{3}^{2}\right),
$$

for $a=1,2$ and the function

$$
f\left(k_{3}\right)=\frac{1}{g k_{3}^{2} \sqrt{k_{3}^{2}+g^{2} k_{3}^{4}}} .
$$

We use the stationary method to approximate the above integrals as

$$
I_{a \lambda}\left(\bar{z}_{0}\right)=f\left(\bar{k}_{3}\right) e^{i \Phi_{1,2 \lambda}\left(\bar{k}_{3}\right)} \int_{-\infty}^{\infty} d k_{3} e^{\frac{i}{2} \Phi^{\prime \prime}\left(\bar{k}_{3}\right)\left(k_{3}-\bar{k}_{3}\right)^{2}},
$$

for a stationary point $\bar{k}_{3}$.

Notice that for $\bar{z}_{0}<0$ there are no stationary points and for $\bar{z}_{0}>0$ we have stationary points only for $I_{1+}\left(\bar{z}_{0}\right)$ and $I_{1-}\left(\bar{z}_{0}\right)$ which are respectively $\bar{k}_{3}=\frac{-\left(z_{3}-\bar{z}_{0}\right)}{2 g \bar{z}_{0}}$ and $\bar{k}_{3}=\frac{\left(z_{3}-\bar{z}_{0}\right)}{2 g \bar{z}_{0}}$ near the light cone. After a straightforward calculation we arrive at

$$
\begin{aligned}
D\left(\bar{z}_{0}, z_{3}\right)= & \sqrt{\frac{2}{\pi}} \frac{\left(g \bar{z}_{0}\right)^{3 / 2}}{\left(z_{3}-\bar{z}_{0}\right)^{3}} \\
& \times\left[\cos \left(\frac{\left(z_{3}-\bar{z}_{0}\right)^{2}}{4 g \bar{z}_{0}}\right)+\sin \left(\frac{\left(z_{3}-\bar{z}_{0}\right)^{2}}{4 g \bar{z}_{0}}\right)\right],
\end{aligned}
$$

where we have used the integrals

$$
\int_{-\infty}^{\infty} d x e^{ \pm i g \bar{z}_{0} x^{2}}=\sqrt{\frac{\pi}{2 g \bar{z}_{0}}}(1 \pm i) .
$$

We observe that the microcausality violation is suppressed by a power $g^{3 / 2}$.

\section{CONCLUSIONS}

Motivated by astrophysical observational tests and their strong limits on the Myers and Pospelov parameter we have considered the possibility to extend the existing treatment for spacelike and lightlike privileged backgrounds. Phenomenological studies based on anisotropies introduced by the spacelike theory are out of the aim of the present work but seems a natural transition. Some works have already been started [19]. The major interest in this work focuses on the consistency of the effective theories that introduce higher dimensional operators. Therefore it shall be considered preliminary work before any phenomenological computation.

It is well known that higher derivative theories can lead to instabilities, negative norm states, and nonunitarity problems to mention some. We have found that the purely spacelike theory is free of the previous issues which strengthen the idea to extend the existing searches for Planck scale phenomena maintaining dimension-5 operators. It also opens the concrete possibility to quantize the theory without the introduction of any cut-off function as required in the timelike case.

To summarize, we have obtained the covariant dispersion relation for the propagation of photons in all 
privileged frames. The timelike, spacelike and lightlike preferred backgrounds have been treated separately for the study of photon kinematic properties. In addition, asymptotes properties of the group velocity, had given us a well idea about causality and stability within the classical theory. For the timelike case we have found runaway solutions above certain values of momenta which suggest the introduction of a cut-off function. The quantization of a model and the implementation of such cut-off function has been carried out in 21]. For the lightlike case we have found additional degrees of freedom with some of them being non analytical. Using the criteria of analyticity we have regarded the lightlike theory as a genuine higher-derivative theory. As further work it would be interesting to identify possible negative energies and to analyze in detail potential negative norm states arising in the theory. Given the similarity with the Chern Simons modification it would be interesting also to study possible extended symmetries and on shell field redefinitions [29]. We have found that the purely spacelike effective field theory is stable and causal while microcausality is highly suppressed. It is therefore appropriate to be quantized. This gives the spacelike Myers and Pospelov theory the possibility to play a role in future searches for Lorentz violation.

\section{Acknowledgments}

I am indebted to H. A. Morales Tecotl, R. Lehnert, M. Cambiaso, J. Gamboa and F. Mendez for valuable comments on this work and encouragement. This work was partially supported by Mexico's National Council of Science and Technology CONACyT-SEP under Grant No. $51132 \mathrm{~F}$ and by Postdoctoral Project DICyT of Usach.
[1] V. A. Kostelecky and S. Samuel, Phys. Rev. D 39, 683 (1989); V. A. Kostelecky and S. Samuel, Phys. Rev. D 40, 1886 (1989).

[2] V. A. Kostelecky and R. Potting, Nucl. Phys. B 359, 545 (1991); V. A. Kostelecky and R. Potting, Phys. Rev. D 51, 3923 (1995).

[3] D. Colladay and V. A. Kostelecky, Phys. Rev. D 55, 6760 (1997); D. Colladay and V. A. Kostelecky, Phys. Rev. D 58, 116002 (1998).

[4] G. Amelino-Camelia, J. R. Ellis, N. E. Mavromatos, D. V. Nanopoulos and S. Sarkar, Nature 393, 763 (1998).

[5] R. Gambini and J. Pullin, Phys. Rev. D 59, 124021 (1999); J. Alfaro, H. A. Morales-Tecotl and L. F. Urrutia, Phys. Rev. Lett. 84, 2318 (2000); J. Alfaro and G. Palma, Phys. Rev. D 67, 083003 (2003).

[6] S. M. Carroll, J. A. Harvey, V. A. Kostelecky, C. D. Lane and T. Okamoto, Phys. Rev. Lett. 87, 141601 (2001); J. Lukierski, H. Ruegg and W. J. Zakrzewski, Annals Phys. 243, 90 (1995); G. Amelino-Camelia and S. Majid, Int. J. Mod. Phys. A 15, 4301 (2000); J. Gamboa, J. Lopez-Sarrion and A. P. Polychronakos, Phys. Lett. B 634, 471 (2006).

[7] G. Amelino-Camelia, Int. J. Mod. Phys. D11, 35 (2002); J. Magueijo and L. Smolin, Phys. Rev. Lett. 88, 190403 (2002); T. Jacobson, S. Liberati and D. Mattingly, Phys. Rev. D 67, 124011 (2003).

[8] V. A. Kostelecky, R. Lehnert and M. J. Perry, Phys. Rev. D 68, 123511 (2003); O. Bertolami, R. Lehnert, R. Potting and A. Ribeiro, Phys. Rev. D 69, 083513 (2004).

[9] R. C. Myers and M. Pospelov, Phys. Rev. Lett. 90 (2003) 211601.

[10] P. A. Bolokhov and M. Pospelov, Phys. Rev. D 77, 025022 (2008).

[11] M. Visser, Phys. Rev. D 80, 025011 (2009).

[12] P. Horava, JHEP 0903, 020 (2009); P. Horava, Phys. Rev. D 79, 084008 (2009).

[13] J. Alfaro and L. F. Urrutia, Phys. Rev. D 81, 025007 (2010).

[14] V. A. Kostelecky and N. Russell, "Data Tables for Lorentz and CPT Violation," arXiv:0801.0287 [hep-ph].
[15] S. M. Carroll, G. B. Field and R. Jackiw, Phys. Rev. D 41, 1231 (1990).

[16] V. A. Kostelecky and M. Mewes, Astrophys. J. 689, L1 (2008); V. A. Kostelecky and M. Mewes, Phys. Rev. D 80 (2009) 015020.

[17] M. Galaverni and G. Sigl, Phys. Rev. Lett. 100, 021102 (2008).

[18] L. Maccione, S. Liberati, A. Celotti and J. G. Kirk, JCAP 0710 (2007) 013; L. Maccione and S. Liberati, JCAP 0808 (2008) 027.

[19] G. Gubitosi, G. Genovese, G. Amelino-Camelia and A. Melchiorri, Phys. Rev. D 82, 024013 (2010).

[20] R. Montemayor and L. F. Urrutia, Phys. Rev. D 72, 045018 (2005); R. Montemayor and L. F. Urrutia, Phys. Lett. B 606, 86 (2005).

[21] C. M. Reyes, L. F. Urrutia and J. D. Vergara, Phys. Rev. D 78, 125011 (2008); C. M. Reyes, L. F. Urrutia and J. D. Vergara, Phys. Lett. B 675, 336 (2009).

[22] T. Mariz, arXiv:1010.5013.

[23] B. Altschul, "Reliable Bounds on Nonrenormalizable Isotropic Lorentz and CPT Violation in QED," arXiv:1010.2779 [hep-ph].

[24] C. Adam and F. R. Klinkhamer, Nucl. Phys. B 607 (2001) 247.

[25] A. A. Andrianov, P. Giacconi and R. Soldati, JHEP 0202 (2002) 030; J. Alfaro, A. A. Andrianov, M. Cambiaso, P. Giacconi and R. Soldati, Phys. Lett. B 639, 586 (2006).

[26] T. C. Cheng, P. M. Ho and M. C. Yeh, Nucl. Phys. B 625, 151 (2002); C. M. Reyes, Phys. Rev. D 80, 105008 (2009).

[27] X. Jaen, J. Llosa and A. Molina, Phys. Rev. D 34, 2302 (1986); J. Z. Simon, Phys. Rev. D 41, 3720 (1990).

[28] V. A. Kostelecky and R. Lehnert, Phys. Rev. D 63 (2001) 065008; R. Lehnert, Phys. Rev. D 68, 085003 (2003).

[29] A. J. Hariton and R. Lehnert, Phys. Lett. A 367, 11 (2007); R. Lehnert, "A mapping between Lorentz-violating and conventional electrodynamics," arXiv:0711.4851 [hep-th]. 